\title{
PLLA biodegradable scaffolds for angiogenesis via Diffusion Induced Phase Separation (DIPS)
}

\author{
V. La Carrubba ${ }^{1}$, F. Carfî Pavia ${ }^{1}$, V. Brucato ${ }^{1}$, S. Piccarolo ${ }^{1}$, G. Ghersi ${ }^{2}$
}

${ }^{1}$ Dipartimento di Ingegneria Chimica dei Processi e dei Materiali, Università di Palermo, Viale delle Scienze, 90128 Palermo, Italy

URL: www.dicpm.unipa.it e-mail: lacarrubba@dicpm.unipa.it

${ }^{2}$ Dipartimento di Biologia Cellulare e dello sviluppo, Università di Palermo, Viale delle Scienze, 90128 Palermo, Italy

URL: http://www.unipa.it/ dipbio/index.htm

e-mail: ghersig@unipa.it

ABSTRACT: A critical obstacle in tissue engineering is the inability to maintain large masses of living cells upon transfer from the in vitro culture conditions into the host in vivo. Capillaries, and the vascular system, are required to supply essential nutrients, including oxygen, remove waste products and provide a biochemical communication "highway". For this reason it is mandatory to manufacture an implantable structure where the process of vessel formation - the angiogenesis - can take place. In this work PLLA scaffolds for vascular tissue engineering were produced by dip-coating via Diffusion Induced Phase Separation (DIPS) technique. The scaffolds, with a vessel-like shape, were obtained by performing a DIPS process around a nylon fibre whose diameter was $700 \mu \mathrm{m}$. The fibre was first immersed into a 4\% PLLA dioxane solution and subsequently immersed into a second bath containing distilled water. The covered fibre was then rinsed in order to remove the excess of dioxane and dried; finally the internal nylon fibre was pulled out so as to obtain a hollow biodegradable PLLA fiber. SEM analysis revealed that the scaffolds have a lumen of ca. $700 \mu \mathrm{m}$. The internal surface is homogeneous with micropores 1-2 $\mu \mathrm{m}$ large. Moreover, a cross section analysis showed an open structure across the thickness of the scaffold walls. A cell culture of endothelial cells was carried out into the as-prepared scaffolds. The result showed that cells are able to grow within the scaffolds and after 3 weeks they begin to form a "primordial" vessel-like structure.

Key words: Tissue engineering, angiogenesis, DIPS

\section{INTRODUCTION}

The production of an engineered tissue starts by the design and the formation of a structure able to support the migration and growth of cells that will originate the new tissue. Those structures (scaffolds) are characterized by a interconnected pore network able to lead, after the degradation, to implanted cells forming a new tissue showing a well integrated structure ${ }^{1}$.

The properties for an ideal scaffold have been identified as: 1) homogenous porosity, to allow cell/ tissue growth as well as the transport of nutrients and removal of metabolic wastes, 2) biodegradability, to match cell/tissue growth in vitro and/or in vivo, 3) suitable surface chemistry for cell attachment, proliferation, and differentiation, 4) pseudo-physiological mechanical properties, and 5) easy of processability in a variety of shapes and sizes $^{2}$. Biodegradable synthetic polymers offer a number of advantages over other materials for developing scaffolds in tissue engineering ${ }^{3}$.
The success of a scaffold after implantation depends on the outcome of a number of complex processes. Initially, after implantation an acute inflammatory response occurs and this is followed by repair processes resulting in wound healing. Simultaneously, the growth of cells into the scaffold and a rapid neo-vascularization of the biomaterial must take place. Therefore implantation of scaffolds where a slow or incomplete vascularization takes place would result in inadequate oxygen and other nutrients supply and eventually hypoxia and cell death $^{4}$.

In the last decade, important advances have been achieved in order to improve the performance of biomaterials to trigger vascular ingrowth (angiogenesis). These approaches are based on the inclusion of angiogenic soluble factors within the matrix or on the engineering of bioactive matrices. However, these attempts are in part limited by the slow endothelial cell (EC) infiltration and by the fast release and biological instability of the angiogenic factors ${ }^{5}$. Another approach is that of combining 
624

different cell types with endothelial cells (coculture) and thus allowing the self-assembly of a capillary-like structure.

In this paper, PLLA scaffolds for vascular tissue engineering, which present a vessel-like shape, were produced by dip-coating via a Diffusion Induced Phase Separation (DIPS) technique. The scaffolds were obtained by performing a DIPS process around a nylon fibre.

Endothelial Cells were successfully cultured within the scaffolds for 3 weeks, thus suggesting the use of those supports as "engineered" vascular grafts.

\section{MATERIALS AND METHODS}

\subsection{Scaffold preparation}

A nylon fibre, whose diameter was $700 \mu \mathrm{m}$, was dip coated with a $4 \% \mathrm{w} / \mathrm{w}$ PLLA - 1.4 dioxane solution at a temperature of $60^{\circ} \mathrm{C}$. The fibre was then extracted from the solution under different pull-out velocities $(2,4,7.2$ and $14.4 \mathrm{~cm} / \mathrm{min})$ as schematized in figure 1; then the fibre was placed in into a second bath containing distilled water at the temperature of $60{ }^{\circ} \mathrm{C}$ for 10 minutes. The covered fibre was successively rinsed with distilled water at room temperature and dried for $36 \mathrm{~h}$ at a temperature of 80 ${ }^{\circ} \mathrm{C}$. Finally the internal nylon fibre was pulled out so as to obtain a hollow biodegradable PLLA fiber 50 $\mathrm{mm}$ long.

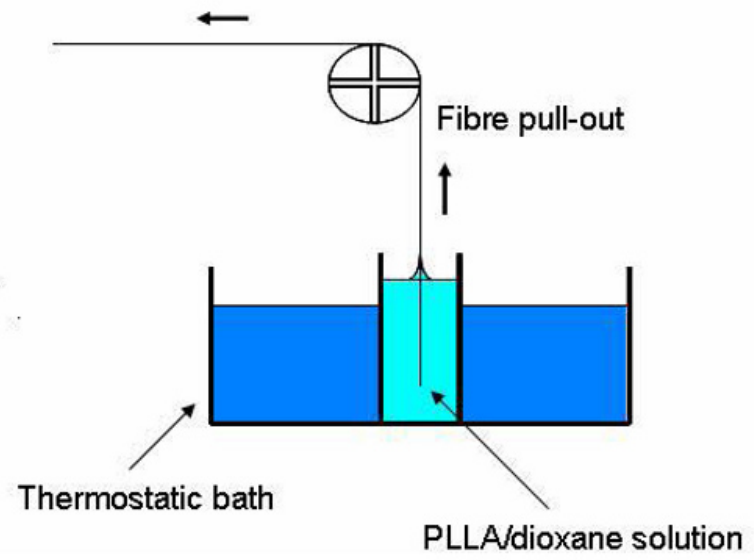

Fig. 1: Experimental set-up for dip coating

\subsection{Cell culture conditions}

The continuous human endothelial cell line ECV304 (IZSBS BS CL 137), purchased from the "Istituto Zooprofilattico" of Brescia, Italy, was cultured in Medium 199 (GIBCO, Grand Island, NY) supplemented with $2 \mathrm{mM}$ L-glutamine, 1 unit $/ \mathrm{ml}$ penicillin, $10 \mu \mathrm{g} / \mathrm{ml}$ streptomycin, 5 units $/ \mathrm{ml}$ heparin and $10 \%$ bovine serum (HyClone, Logan, UT).

ECV304 cell were seeded inside to PLLA scaffold tube pre-treated with acidic $\left(0,02 \mathrm{~N} \mathrm{CH}_{3} \mathrm{COOH}\right)$ type-I collagen fibrils solution, buffered with complete media ( 2 wash); at $1,2 \times 10^{7}$ cell $/ \mathrm{ml}(200$ $\mu 1)$. Media were exchanged every $2 / 3$ days.

\subsection{Cells observation}

Scaffolds were fixed 10' r.t. in culture media buffer supplemented with 4\% gluteraldehyde; washed 3 times in PBS containing $\mathrm{Ca}^{++}$and $\mathrm{Mg}^{++}$. Tubes were cut across the transverse direction in smaller pieces about $8-10 \mathrm{~mm}$ long, longitudinally opened and analyzed by different staining method. Some were directly stained with a solution of $0,2 \%$ Comessie Blue R-250 in a ternary solution $\mathrm{CH}_{3} \mathrm{OH}: \mathrm{CH}_{3} \mathrm{COOH}: \mathrm{H}_{2} \mathrm{O}(5: 1: 5)$ for 3 minutes and washed in $\mathrm{H}_{2} \mathrm{O}$ (at least 5 times). Some other samples were incubated in a solution containing FITC-Phalloidin $(0,006 \%$ - Sigma $)$ and Ethidium bromide $(10 \mu \mathrm{g} / \mathrm{ml}$ - Sigma $) 2 \mathrm{hr}$ at $4^{\circ} \mathrm{C}$; than washed 5 times in PBS containing $\mathrm{Ca}^{++}$and $\mathrm{Mg}^{++}$ and mounted with mounting buffer (glycerol $80 \%$ plus $\mathrm{NaN}_{3} 0,02 \%$ in Tris buffer $0,5 \mathrm{M} \mathrm{pH} 7,4$ ).

Prepared samples were observed under an Olympus Floview FV300 laser confocal microscopy $(2-\mu \mathrm{m}$ laser sections).

For the Scanning Electron Microscopy (SEM) observation, the samples were fixed as described above, rinsed in PBS $1 \mathrm{X}$ and dehydrated in increasing concentrations of ethanol. Dehydrated samples were gold sputter coated and observed using a PHILIPS 505 SEM.

\section{RESULTS}

\subsection{Scaffolds morphology}

A SEM analysis revealed that the as-prepared scaffolds present a lumen of $\sim 500-600 \mu \mathrm{m}$ (see figure 2). The internal surface is homogeneous with micropores whose average diameter is $1-2 \mu \mathrm{m}$ (see figure 3).

Polymer concentration, solution viscosity and density as well as fibre drawing speed are expected to play a crucial role in determining and controlling the scaffold wall thickness.

In figures 4, 5 and 6, SEM images of the cross section of scaffolds prepared under different fibre drawing speeds are showed. It is easy to notice that the scaffolds prepared under larger pull-out 
velocities present a thicker wall.

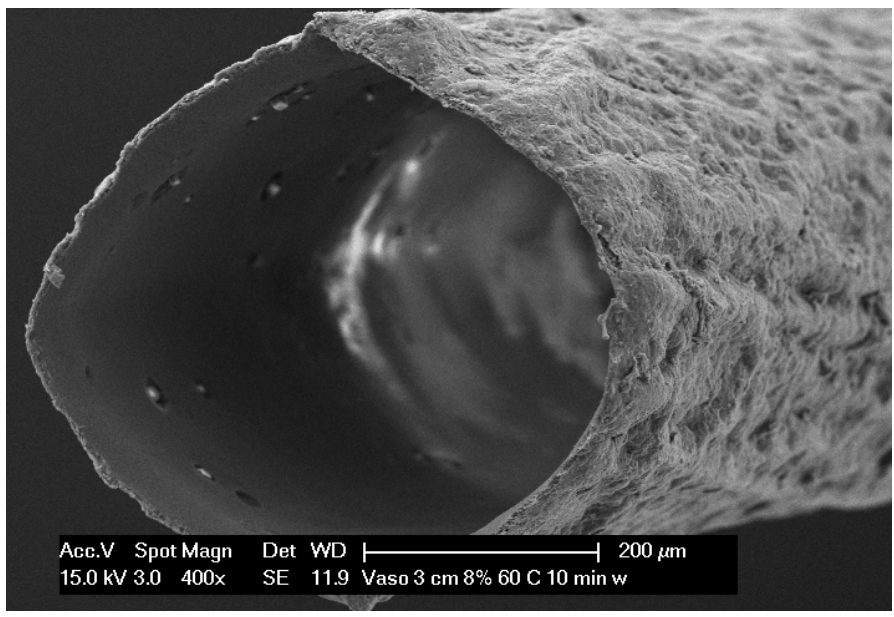

Fig. 2: SEM image of the cross section of the scaffold for vascular tissue engineering.

An accurate analysis of the scaffold wall revealed that wall-thickness exponentially depends on the speed at which the fibre is drawn from the PLLA dioxane solution (see figure 7 , reporting wall thickness on a logarithmic scale as a function of fibre pull-out velocity).

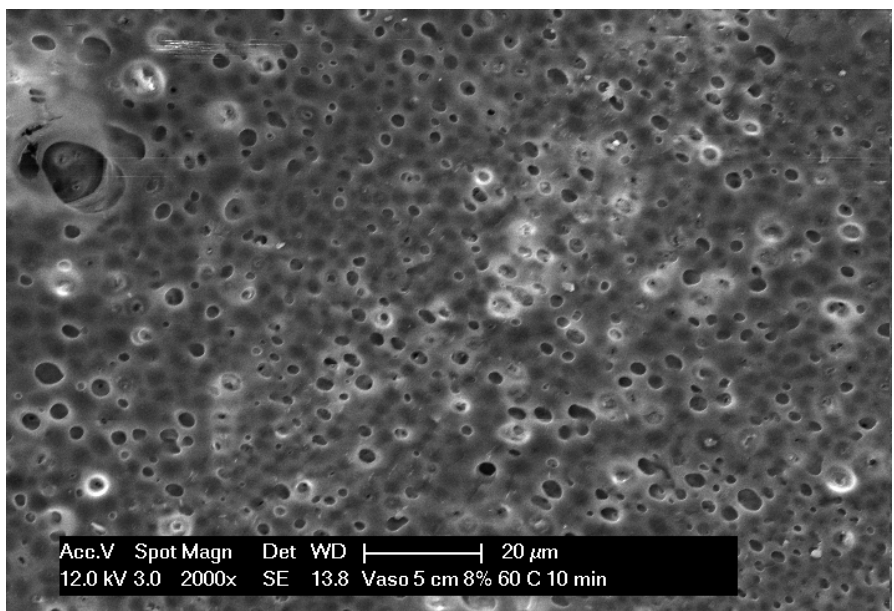

Fig. 3: SEM image of the internal surface of the scaffold

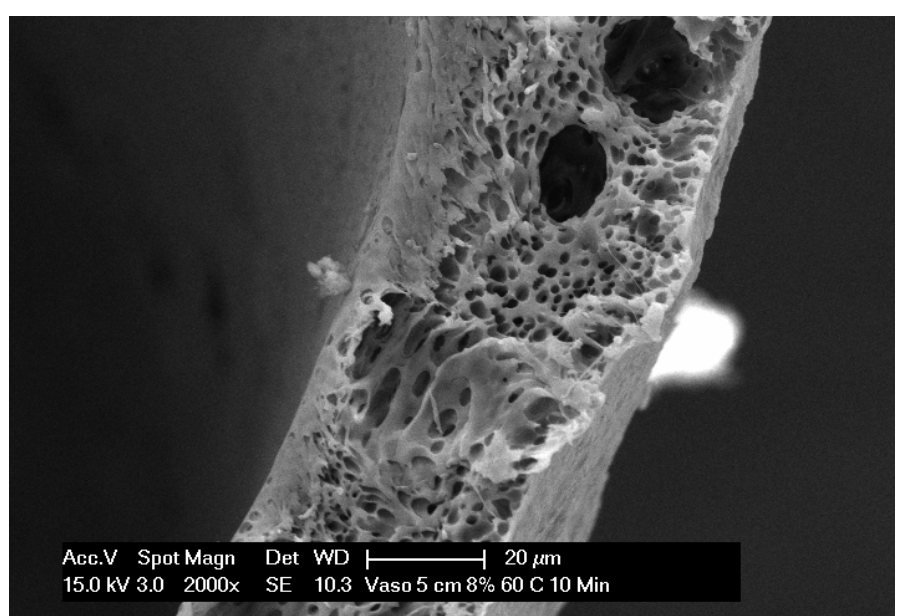

Fig. 4: SEM image of the cross section of a scaffold prepared by pulling out the nylon fibre from the PLLA/dioxane solution at $14.4 \mathrm{~cm} / \mathrm{min}$.

Finally, from these images is possible to observe an open structure along the thickness of the scaffold walls, with a porosity almost uniformly distributed, except for a very thin layer (few $\mu \mathrm{m}$ thick) close to the outer and inner surfaces.

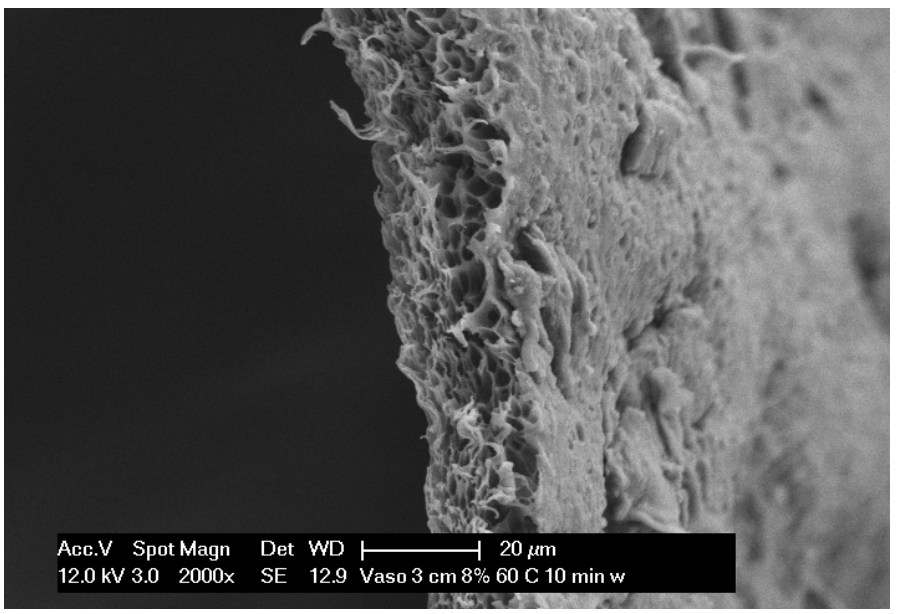

Fig. 5: SEM image of the cross section of a scaffold prepared by pulling out the nylon fibre from the PLLA/dioxane solution at $7.2 \mathrm{~cm} / \mathrm{min}$.

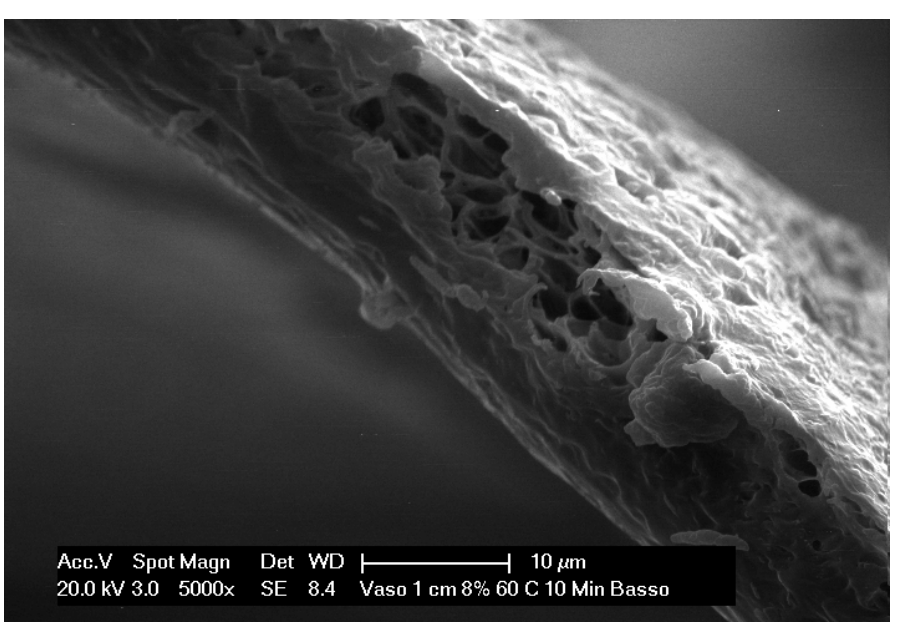

Fig. 6: SEM image of the cross section of a scaffold prepared by pulling out the nylon fibre from the PLLA/dioxane solution at $2.4 \mathrm{~cm} / \mathrm{min}$.

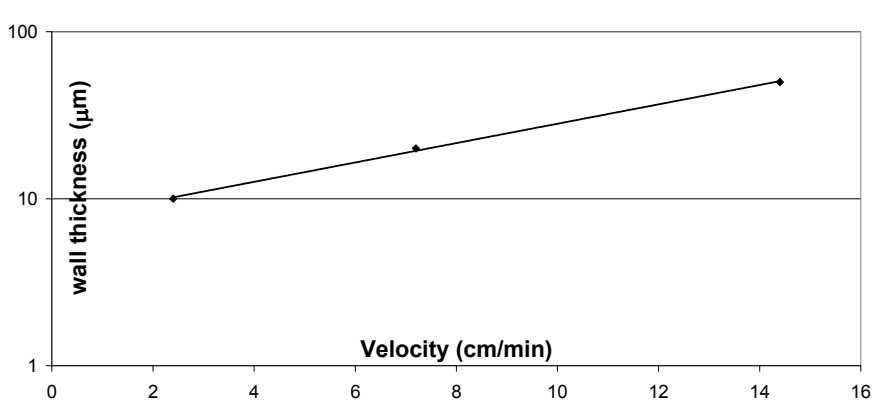

Fig. 7: Wall thickness versus fibre pull-out velocity

\subsection{Cells cultures}

A cell culture inside these scaffold was carried out, by using endothelial cells (EC), which are the solely components of capillary bed and are the first to form during embryonic development. Figure 8 illustrates a laser confocal microscope image of a cross section of a scaffold with EC grown 3 weeks. The part 
626

coloured in red are the nuclei of the cells, while the areas in green indicate the actin, a ubiquitary protein. The image clearly shows that after 21 days the internal lumen of the scaffold is totally covered by EC, which have organized into a real vessel structure. This result was confirmed by the SEM analysis of the same section (see figure 9).

As a matter of fact, the SEM image reported in fig. 9 should be compared to fig. 3 , where the microporous internal surface of the scaffold is shown in the absence of EC. It is easy to notice in fig. 9 that EC covered completely the scaffold internal surface, as the micropores are not anymore visible.

These experiment suggests that the scaffold produced could be usefully employed in vascular tissue engineering.

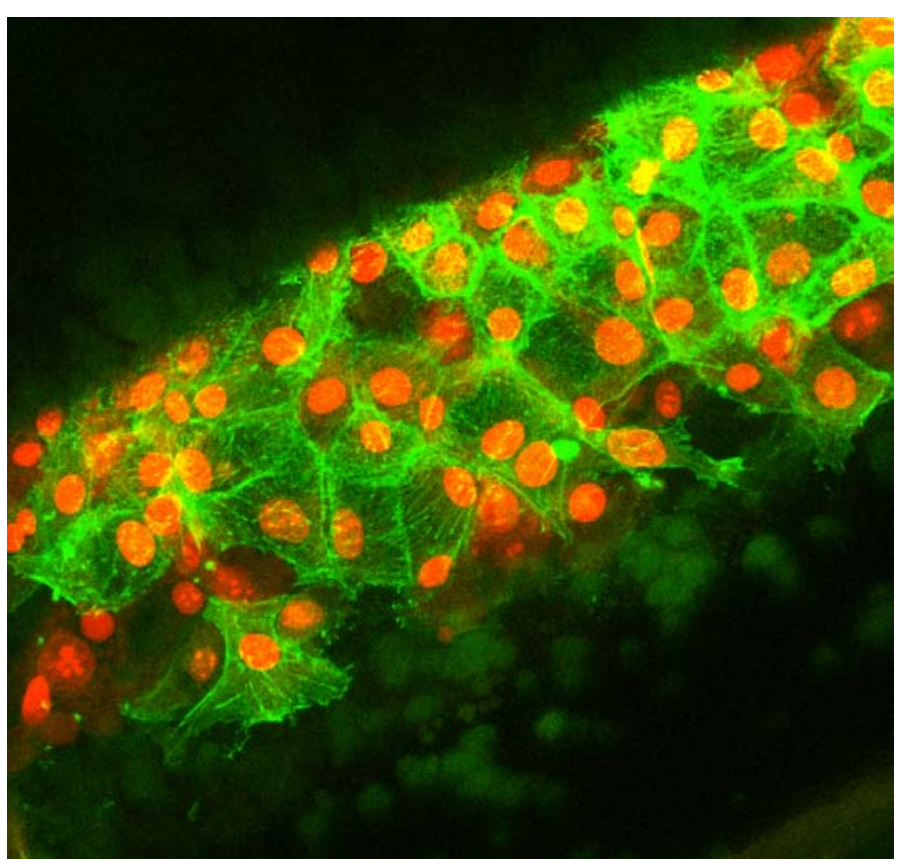

Fig. 8: Confocal microscope image of the EC grown inside the scaffold for 21 days.

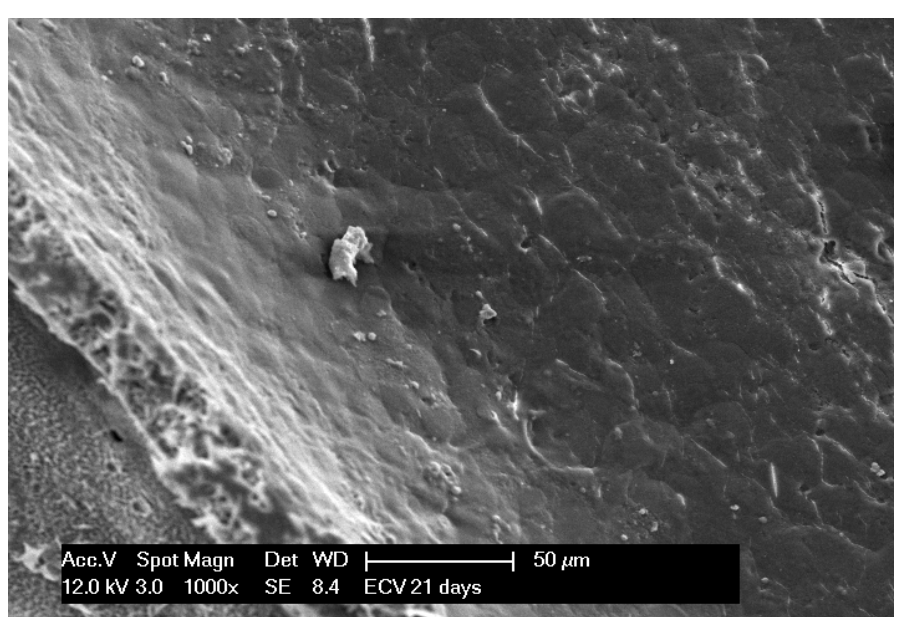

Fig. 9: SEM image of the EC grown inside the scaffold for 21 days.
PLLA scaffolds with a vessel-like shape were dipcoated by means of a Diffusion Induced Phase Separation (DIPS) method. The scaffolds obtained present a lumen of $\sim 500-600 \mu \mathrm{m}$, a relatively large internal porosity and an open structure. Moreover, wall thickness can be modified simply varying a simple experimental parameter, the pull-out velocity from the PLLA/dioxane bath.

These features suggest the possibility of a successful use in the field of the vascular tissue engineering, as confirmed by the endothelial cell culture experiments. The results showed that after 3 weeks endothelial cells not only grew inside the scaffold, but also they organized themselves into a real vessel structure.

A modelling of the dip-coating is in progress, together with an experimental campaign concerning the biodegradation kinetics in relation with the processing parameters.

\section{REFERENCES}

1. C Schugens, V Maquet, R Grandfils, R Jerome, P Teyssie, Biodegradable and macroporous polylactide implants for cell transplantation: 1. Preparation of macroporous polylactide supports by solid-liquid phase separation. Polymer 37 (1996) 1027-1038.

2. F Coueta, N Rajana and D Mantovani, Macromolecular Biomaterials for Scaffold-Based Vascular Tissue Engineering. Macromol. Biosci. 7 (2007) 701-718

3. PA Gunatillake and R Adhikari, Biodegradable synthetic polymers for tissue engineering. European Cells and Materials 5 (2003) 1-16

4. RE Unger, A Sartoris, K Peters, A Motta, C Migliaresi, M Kunkel, U Bulnheim, J Rychly and CJ Kirkpatrick, Tissuelike self-assembly in cocultures of endothelial cells and osteoblasts and the formation of microcapillary-like structures on three-dimensional porous biomaterials. Biomaterials 28 (2007) 3965-3976

5. C Borselli, O Oliviero, S Battista, L Ambrosio, PA. Netti, Induction of directional sprouting angiogenesis by matrix gradients. J biomed mat res, part A 80A (2007) 297-305 